\title{
Percepción de los coordinadores, tutores y alumnos en relación a la tutoría como estrategia de enseñanza aprendizaje
}

\author{
Coordinators, tutors, and alumni's perception \\ on tutoring as teaching-learning stategy.
}

Lic. Madai Silva González* Mtra. Margarita Cárdenas Jiménez**

*Pasante de la Licenciatura en Enfermería incorporada en el proyecto PAPIIT IN-312503 como opción de titulación

${ }^{\star *}$ Responsable del proyecto y Asesora

\section{Resumen}

El presente trabajo es producto de la investigación: "Evaluación Curricular de las Especialidades en Enfermería", que tiene como propósito conocer los factores que inciden en el proceso del aprendizaje especializado el cual permita reestructurar por medio de la tutoría la práctica profesional del especialista en enfermería y mejorar la calidad del aprendizaje transformando la práctica.

Una de las vertientes de análisis que se realizo fue: identificar el significado que le atribuyen, las coordinadoras, los tutores y los alumnos, a la tutoría desde la información vertida en las entrevistas registradas.

Objetivo: Determinar los significados relacionados con la tutoría desde la perspectiva de las coordinadoras, tutores y alumnos (as) de las especialidades a través del análisis de su propia opinión, para establecer la importancia que tiene la tutoría en el proceso de aprendizaje de los futuros especialista.
Metodología: Se considero la información vertida en las entrevistas a profundidad registradas y se realizó el análisis de los significados relacionados con la tutoría los cuales se confrontaron con los elementos teóricos relacionados con dicha temática.

Resultados: Se encontró que el rol que juega el tutor con el alumno ejerciendo la TUTORIA es un proceso significativo desde la enseñanza aprendizaje hasta su total formación como especialista, siendo la tutoría una base fundamental . Conclusiones: La tutoría es una actividad fundamental dentro del proceso de formación de la especialidad ya que permite el desarrollo integral de los alumnos.

Palabras Clave: Tutoría, Especialidad, Enfermería, Rol.

\section{Abstract}

Seeking categorical components involved in specialized teaching- learning processes to improve tutoring as a qualityadding option, this analysis is a sub-product of the research paper: "nursing specialties curricula assessment" Objective: to gather concepts related to tutoring from the perspectives of the coordinators, tutors, and alumni of the nursing specialties in order to determine its relevance, importance, and meaning. Methodology, tutors play was highly significant. Conclusions: promoting integrality, tutoring is a fundamental activity among nursing specialty forming processes.

Key words: nursing, specialty, tutoring, roll 


\section{INTRODUCCIÓN}

El presente trabajo es producto de la investigación titulada: "Evaluación curricular de las Especialidades en enfermería"1, cuyo propósito fue conocer los factores que inciden en el proceso del aprendizaje especializado, siendo la motivación principal de la investigación el ámbito académico de las Especializaciones en Enfermería de la Escuela Nacional de Enfermería y Obstetricia de la UNAM y es la evaluación del currículum en sus formas específicas para llevar a cabo los aprendizajes, lo que condujo a la construcción del objeto de estudio a partir de la problematización del campo de estudio, en donde el currículum dirige a los maestros y alumnos a establecer la relación entre poder y conocimiento desde varias líneas de interpretación: el curriculum dominante, en donde el conocimiento es frecuentemente aislado de la cuestión del poder y es generalmente tratado de manera técnica, es decir desde la racionalidad técnica, el cual es visto en términos instrumentales como algo a ser dominado. Esta investigación tiene un fin de mejorar la calidad del aprendizaje transformando la práctica profesional en enfermería.

Las etapas en las que se llevó a cabo la metodología propuesta fue la siguiente:

I.- El análisis curricular a partir de la Hermenéutica significa que lo ubica en el contexto sociopolítico en el que fue diseñado el proyecto educativo, para ello se realizó el análisis del discurso de la fundamentación disciplinar y pedagógica; así como el análisis de la congruencia interna y externa del Plan desde la Teoría Crítica de la educación: Procesos dialógicos, comunicación y praxis. En este análisis se consideraron los siguientes aspectos: Perfil académico profesional, Objetivos y contenidos de las actividades académicas, organización curricular, programas académicos, evaluación, fundamentación disciplinar pedagógica de cada orientación del Plan Único de Especialización.

II.- El trabajo de Campo consistió en tres fases: interpretación de los resultados obtenidos en la observación y entrevista, identificación de categorías significativas, la búsqueda de nuevos significados en las observaciones y entrevistas subsecuentes y las interpretaciones a la luz de las categorías de análisis definidas. Así las entrevistas a profundidad, fueron previamente ejercitadas para analizar aciertos y errores en las mismas y la observación participante se realizó a los alumnos seleccionados durante su práctica clínica o comunitaria incluyendo el trabajo áulico. Las entrevistas se efectuaron con los actores involucrados en el proceso de formación: coordinadoras, tutores y estudiantes, fueron grabadas en audio, transcritas a través del programa digital voice editor e interpretadas a través del análisis del discurso, categorización del mismo y por último identificación de significados.

III.- El análisis de los resultados se efectuó paralelamente con la etapa anterior por lo que aquí se observaron los resultados a través de las categorías establecidas, revisándose la interacción docente, la participación crítica desarrollada, la acción comunicativa y la calidad de los resultados obtenidos en el proceso de indagación, en el contexto de un taller permanente, espacio de reflexión acción construcción, desarrollado durante la investigación.

IV.- Presentación del Informe de Resultados:

se está realizando a través de la triangulación por métodos afines la cual consiste en la combinación de dos o más procedimientos similares de recolección de datos (la observación, la entrevista a profundidad y el análisis hermenéutico del plan de estudios), cuyos datos son codificados y analizados por separado que comparados entre sí permitan establecer conclusiones.

V.- Redacción y presentación del informe final.

Se han analizado diferentes vertientes de los resultados obtenidos en dicha investigación, el presente, es uno más, por tanto, en un primer momento en mi experiencia como becaria del proyecto, fue identificar el significado que le atribuyen las coordinadoras, los tutores y los alumnos, a la tutoría desde la información vertida en las entrevistas registradas.

El objetivo planteado para el desarrollo de este trabajo es considerar la importancia que tiene la tutoría en el proceso de aprendizaje de los futuros especialistas, desde la opinión de los participantes. Cuyo objetivo se deriva del objetivo del proyecto general.

Para el análisis de los significados relacionados con los participantes, estos se confrontaron con los elementos teóricos relacionados con la tutoría.

\section{ASPECTOS ÉTICOS.}

Esta investigación no afectó en ningún momento la credibilidad, ni atentó contra la individualidad de los sujetos de estudio, en tanto que la información recopilada fue totalmente anónima así mismo se tomó en cuenta lo siguiente: Consentimiento informado y por escrito, confidencialidad de la información, y su utilización considerando el derecho de propiedad de los informantes, intimidad en la realización de las entrevistas, respeto a la dignidad de los informantes, riesgos y beneficios de la investigación entre otros. 


\section{RESULTADOS}

Los resultados obtenidos fueron a través de las entrevistas aplicadas a los siguientes actores involucrados: una coordinadora de la especialización como informante clave, un docente de actividad académica o un tutor clínico, un alumno por cada una de las especializaciones siguientes: Enfermería del Adulto en Estado Crítico, Cardiovascular, Enfermería en Salud Pública y Enfermería Infantil, sumando un total de 16 personas. A continación se presentan los cuadros referentes a las citadas entrevistas.

\section{Cuadro 1}

\section{Opiniones de las coordinadoras en relación a la tutoría}

"Se ejerce la tutoría con los alumnos platicando con ellos cuestionando sobre las experiencias que tienen en los servicios, que aprendieron y que dudas tienen..."

"La responsabilidad como tutor es proveer el conocimiento al alumno".
"La tutoría siempre enseña tanto al alumno como al tutor dejando en cada uno enseñanza continua, siendo esta la base del posgrado".

"La tarea de la tutoría es desarrollar habilidades y estrategias en los alumnos, y ver cómo vamos a guiar al alumno".

"La tutoría es una estrategia metodológica en el posgrado es un modelo que les impacta a los alumnos siendo la función principal de un tutor llevar al alumno al aprendizaje ya sea tanto en el aspecto teórico como en el aspecto práctico".

Fuente: entrevistas a profundidad.

\section{Opiniones de los tutores en relación a la tutoría}

"La tutoría implica guiar al alumno, tanto a nivel de conocimientos, como a nivel de práctica".

"Las principales funciones de la tutoría son: proporcionar actitudes, conocimientos, experiencia con los alumnos, guiarlos en lo que es el aspecto clínico, orientarlos en todos los trabajos, revisar y calificar los trabajos, evaluar todo proceso de aprendizaje que se está dando, tanto a nivel de ellos como en nosotros".
"La tutoría es algo esencial para que nosotros podamos llevar a cabo la especialidad de enfermería y es algo valioso y fundamental".

"La tutoría es una necesidad porque lo vemos con los grupos, es una estrategia de aprendizaje ya que entra dentro de las estrategias que se utiliza para la enseñanza del proceso enseñanza - aprendizaje, de los tutores depende el aprendizaje de los alumnos". 


\section{Opiniones de los alumnos en relación a la tutoría}

"Lo que es el sistema tutorial yo alumno me acerco al profesor para que me asesore en el momento en el que yo lo necesito, o sea, no que él me necesite, que él me tenga que decir no pues tú haces esto o que tienes que hacer esto".
"Nos permite que conozca mas sobre por ejemplo procesos patológicos y que la tutora te encamine a que si está bien realizado o algunos aspectos que manejan por ejemplo otras áreas todo este lo mejor hecho para beneficio del paciente".
"Es saber que dudas tengo, que estoy haciendo bien, o haciendo mal o uno como alumno se acerque y pregunte al tutor, sabes que no sé cómo hacer esto, se que se hace así, pero no sé si lo estoy haciendo bien, enséñame, y yo creo que la obligación del tutor es saber, ya que el hecho de que uno no lo practique tanto pierde ciertas habilidades".

"El ideal de tutor para que precisamente provocar el aprendizaje ser una persona consciente, culta, que me sepa escuchar, debe de comprender, pero también sin perder el papel de que él tiene el mando".
"Con la tutoría te vas llevando a ser un buen caso sobre lo que es la practica a lo que te dieron previamente pero ya con el paciente ya te cambia algunas cosas entonces el hecho de que tengamos a alguien que nos esté identificando o que nos esté ayudando a identificar esas necesidades y que tenga más conocimientos que nosotros entonces tendríamos que desarrollarlo mejor".

Fuente: entrevistas a profundidad.

\section{DISCUSIÓN}

Algunas coordinadoras consideran que la forma en que se ejerce la tutoría es "platicando" cuando esta estrategia de Enseñanza aprendizaje debe ser intencionada y planeada por el tutor utilizando diversas formas de comunicación, así mismo otra considera que la acción tutorial es proveer de conocimientos al alumno marcando una relación unidireccional y no dialógica como es la pretensión de la tutoría.

Por otra parte, es notoria la opinión de los tutores donde uno(a) de ellos(as) hace una descripción detallada de las implicaciones de la tutoría, y el resto se concretan a resaltar la importancia que tiene en las especialidades. En contraste, los alumnos al hacer referencia sobre la tutoría señalan puntualmente los requisitos que debe de tener un tutor; "es su obligación saber" "provocar el aprendizaje" "ser una persona consciente" "culta" "que me sepa escuchar" "debe de comprender" "nos permite que conozca más" "que nos este identificando esas necesidades de aprendizaje" "que tenga más conocimientos" "que ofrezca todos sus conocimientos". De lo antes expuesto, se puede observar que tanto coordinadoras (es) como tutoras (es) su visión en cuanto a la tutoría está más relacionada a la importancia para el aprendizaje de las especialidades y los alumnos por su parte, se enfocan hacia el perfil que debe tener el tutor.
"Que sea una persona que ofrezca todos sus conocimientos sin olvidarse que hay que tener en cuenta los aspectos teóricos previos y en ayudarme a desarrollar ese proceso de aprendizaje el cual me da la oportunidad de contar con más información en este periodo semestral".
Consideran que la tutoría debe ser dada en el momento en que el alumno lo necesite y no en que el tutor lo quiera dar, así el alumno pretende que el tutor valore los avances, las dudas, que es lo que está haciendo, si se hacen bien o mal las cosas, de que sea una persona consciente y que sepa escuchar. Algunas ocasiones la tutoría se enfoca hacia el aprendizaje de los procesos patológicos más que los aspectos disciplinares de la enfermería, sin embargo un análisis permanente de la tutoría ejercida entre tutores y alumno puede reorientarla.

Se puede afirmar que un aspecto importante donde coinciden los entrevistados es que dentro de la formación del especialista es la estrategia de enseñanza aprendizaje de las especialidades. Si partimos de que la tutoría es una acción coordinada por un docente capaz de dar y de fomentar la capacidad crítica y creadora en el rendimiento académico y el desarrollo social y personal de los estudiantes siendo un experto en consultas no estructuradas, en las que el asesor esclarece las dudas del alumno cuando lo considere necesario (2). La tutoría es un proceso de acompañamiento durante la formación de los estudiantes en la especialidad que se concreta mediante la atención personalizada a un alumno o a un grupo reducido, por parte de un profesor capacitado competente y formado para dicha función(3). 
Entonces el tutor debe emprender acciones dialógicas y horizontales que permitan la discusión de las situaciones y problemas vividos por los estudiantes, Si los estudiantes son concebidos simplemente como receptores y receptáculos del acervo y de saberes profesionales y no se les permite, la reconstrucción personal se les mantendrá alineados de sí mismos y de sus potencialidades. Es así como el tutor juega un papel importante en la especialidad ya que es capaz de elevar la calidad de los estudiantes en el ámbito de los valores, y actitudes siendo este responsable, confiable, sociable, comunicativo y de espíritu motivador y conciliador. La tutoría implica la asesoría académica, en la que el asesor esclarece las dudas o ayuda en la planeación de un programa, subsana las desventajas que la enseñanza tradicional ha tenido respecto a la atención del ritmo de aprendizaje y de motivación de los estudiantes logrando así los objetivos deseados conforme a las actividades académicas. La enseñanza tutorial representa un proceso semejante a la relación maestro-alumno, en la que gradualmente ayuda al alumno a eliminar errores, así Alcántara Santuario(4) menciona en este sentido que: "la tutoría es un método de enseñanza en donde es más fácil que dos mentes trabajen juntas en un problema, que si una sola lo hiciera por separado".

Uno de los aspectos más importantes en el desarrollo como especialistas destaca la tutoría como un papel fundamental dentro de la formación académica y siendo un proceso importante de aprendizaje, enseñando al alumno de la responsabilidad de su propio aprendizaje, la tutoría como estrategia educativa, produce consecuencias directas de gran importancia en el proceso educativo, consecuencias que tienen que ser ponderadas ya que la formación de los alumnos en la especialidad en enfermería implica establecer relaciones entre el aprendizaje y la enseñanza.

La tutoría implica un conjunto de habilidades que favorecen el acompañamiento del alumno en el proceso educativo. Por ello, el tutor debe poseer una actitud ética y empática, mediante un esfuerzo permanente de comunicación en una actuación siempre responsable, atendiendo los compromisos con toda puntualidad en un marco de respeto y confidencialidad. Así la misión de la tutoría es el de proveer orientación sistemática al estudiante, desplegada a lo largo del proceso educativo, desarrollando una gran capacidad en el tutor estimulando las capacidades para el aprendizaje y el desempeño del alumno (5).

Podemos concluir y afirmar que el tutor es parte fundamental de la formación del especialista en enfermería siendo esta pieza clave para la formación del alumno.

\section{CONCLUSIONES}

1. Los entrevistados coinciden que la tutoría es la base fundamental de la formación del especialista en enfermería.

2. No se precisa la concepción de tutoría y sus implicaciones en la mayoría de las (los) coordinadores (as) y tutoras(es).

3. Solo una tutora describe ampliamente las implicaciones que tiene la tutoría en el proceso de aprendizaje de los alumnos.

4. Los alumnos conciben la tutoría a partir del perfil que esperan de ellos.

5. El ideal tutor para el alumno es aquella persona que ofrece todos sus conocimientos sin olvidarse de los aspectos teóricos y que ayuda a desarrollar un proceso de aprendizaje en el especialista.

6. La tutoría según los alumnos debe ser dada en el momento en que él lo necesite y no en que el tutor lo quiera dar.

7. El alumno pretende que el tutor valore los avances, las dudas que tiene, que es lo que está haciendo y si lo hace bien o mal.

\section{REFERENCIAS BIBLIOGRÁFICAS}

1. Cárdenas J.M. Evaluación Curricular de las Especialidades en Enfermería, ENEO-UNAM 2003

2. Moran P.I. El Sistema de la Enseñanza tutoral en Enfermería documento interno, ENEO-UNAM Febrero del 2000

3. ANUIES. El sistema Tutorial, en: Problemas institucionales de tutorías. Una propuesta de la ANUIES para su organización y funcionamiento en las Instituciones de Educación superior". Colección Biblioteca de la Educación superior. Serie Investigaciones. $2^{\mathrm{a}}$ ed. México, D.F., 2002. pp 41-46

4. Alcántara S. A. Consideraciones sobre la tutoría en la enseñanza universitaria, en Perfiles Educativos, No. 49-50, JulioDiciembre 1990. pp. 51-55

5. Ostiguín M.R.M. y Rivas R.B.N Percepción de los Alumnos del Posgrado en Enfermería sobre el Sistema Tutoral ENEOUNAM, México 2000

6. Lipson G.J. Asuntos Éticos en la etnografía en Asuntos Críticos en los métodos de investigación cualitativa, Universidad de Antioquia Columnia 2003 p. 391

7. Balseiro A.L. Investigación en Enfermería editorial Prado México D.F 1991 pp. 125-126

\section{DIRECCIÓN PARA CORRESPONDENCIA}

Mtra. Margarita Cárdenas J.: cardenas950@hotmail.com 